\title{
Preliminary study of the influence of DED on carbon radiation and transport in the TEXTOR tokamak
}

\author{
G. Telesca ${ }^{\mathrm{a}, *}, \mathrm{~K}$. Crombé ${ }^{\mathrm{a}}, \mathrm{M}$. Tokar ${ }^{\mathrm{b}}$, B. Unterberg ${ }^{\mathrm{b}}$, \\ G. Verdoolaege ${ }^{\mathrm{a}}, \mathrm{R}$. Zagorski ${ }^{\mathrm{c}}, \mathrm{G}$. Van Oost ${ }^{\mathrm{a}}$ \\ a Department of Applied Physics, Ghent University, Rozier 44, B-9000 Gent, Belgium \\ b Institut fuer Plasmaphysik, Forschungszentrum Juelich GmbH, EURATOM Association, D-52425 Juelich, Germany \\ c Institute of Plasma Physics and Laser Microfusion, P.O. Box 49, 00-908 Warsaw, Poland
}

\begin{abstract}
Changes on transport and radiation properties of intrinsic carbon under the influence of the Dynamic Ergodic Divertor depend on the modal composition of the induced error field. For 12/4 mode operation, significant carbon screening is not observed up to half of the nominal DED maximum current achieved so far. On the contrary, for 3/ 1 DED operation the very efficient mechanism of expulsion of highly ionized carbon ions, observed to occur locally at the foot prints of the $3 / 1$ helical structure and explained in terms of frictional forces, leads to a global carbon decontamination of the central plasma. This result has been established using a new multi-chord diagnostic, which allows to distinguish global from local carbon behavior.
\end{abstract}

(C) 2004 Elsevier B.V. All rights reserved.

\section{Introduction}

In this paper experimental data on carbon behavior under the influence of the dynamic ergodic divertor, DED, on TEXTOR [1] are reported and discussed. The DED is mainly intended to optimize heat and particle exhaust through the formation of a magnetic divertor and of a stochastic layer at the plasma edge. The study of the response of intrinsic carbon - in terms of transport and radiation properties - to a change in the

\footnotetext{
* Corresponding author. Tel.: +49 2461 615174; fax: +49 2461613331.

E-mail address: g.telesca@fz-juelich.de (G. Telesca).
}

edge field structure can provide a key element to assess the viability of the DED concept to reactor grade experiments. The data refer to the first experiments with DED operating at $m / n=12 / 4$ and at $m / n=3 / 1$. For the $12 / 4$ mode, engineering problems have limited so far the level of the currents in the DED coils and by consequence the level of the associated error field, which at present reaches about half of its nominal value, see Ref. [2]. Since the decay length of the error field is a strong function of $m$ and it is short for $m=12$ [3], the external field in $12 / 4$ operation affects only the very edge of the plasma and its action is marginal if the DED currents are only half of their maximum nominal value. In this sense, the data reported for the $12 / 4$ mode have to be considered very preliminary. On the other hand, the global 
diagnostic capability of TEXTOR during the $12 / 4$ campaign and partly also during the subsequent $3 / 1 \mathrm{cam}$ paign, was modest (the 12/4 campaign was the first experimental campaign after a long shut down). For example, the Electron Cyclotron Emission diagnostic for the measurement of the electron temperature profile was not routinely operational during the $12 / 4$ mode experiments as well as $Z_{\text {eff }}$ and the charge exchange recombination spectroscopy, CXRS. The aim of the present work is to establish criteria useful for the study of the effects of the DED on transport and radiation of the intrinsic carbon. The specific diagnostic used for this study is a new eight channel spectroscopic carbon diagnostic, which has been designed to monitor the change in carbon flux and density under the action of DED. Indeed, as experimentally shown by the Ergodic Divertor in the tokamak Tore Supra [4] and theoretically predicted [5], at the onset of the perturbing radial field one should expect the increase of impurity screening as well as the increase of the ratio of power radiated to impurity density in the plasma core. After a short description of the new carbon diagnostic (Section 2), the data are presented in Section 3 and discussed in Section 4.

\section{UV and VUV carbon diagnostics}

The brilliances of a CIII line and of a CV line in the UV range (at $229.7 \mathrm{~nm}$ and at $227.1 \mathrm{~nm}$, respectively), are detected simultaneously along each of the eight lines of sight (from channel 2-9; channel 1 is not yet operational) shown in Fig. 1 [6]. Four channels (N. 2,3,4,5) intercept the divertor plates in the high field side where the DED coils are located, the other four can provide information of carbon behavior far from carbon sources. According to the design of the diagnostic, all the 16 (18) signals will be detected simultaneously at a sampling rate of $20 \mathrm{kHz}$. At present, however, the diagnostic can operate only at reduced performance that is the CIII and CV signals are detected simultaneously along each of the eight channels but with a time resolution of $100 \mathrm{~ms}$. Unfortunately, a technical problem prevented so far the accomplishment of a reliable relative calibration of the eight cannels. This means that the relative intensities of the 16 signals are not related to the relative photon fluxes. By consequence, we have to limit ourselves to the comparison of the time evolution of the 16 signals, each independently of the others. A second operational mode of the diagnostic consists in detecting continuously in time the CIII and CV signals from only one out of the eight channels, at the cost, however, of loosing information from the other seven channels. In the toroidal sector of our diagnostic, for 12/4 mode of operation the foot prints of the strikes zones are located outside the small areas (a few square centimeters each)

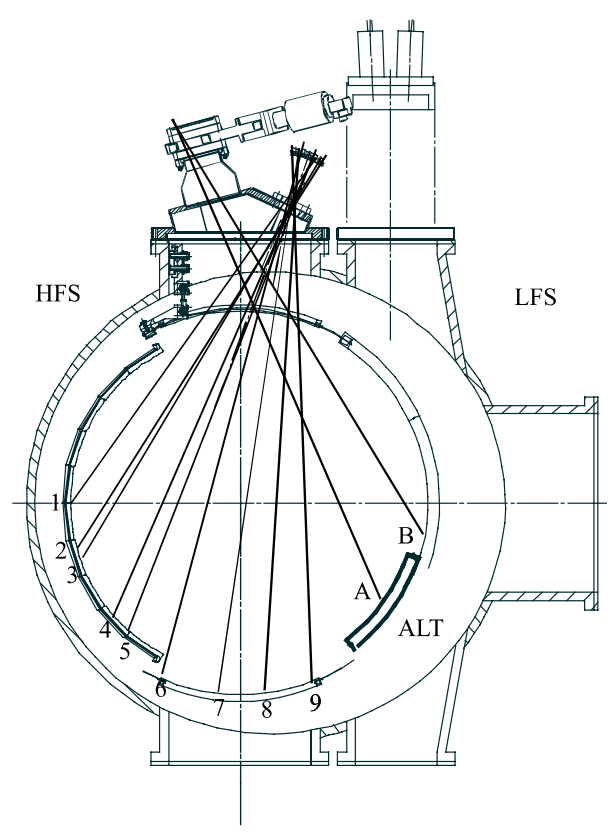

Fig. 1. Lines of sight of the UV carbon diagnostic. Channel 1 is not yet operational.

of the divertor plates intercepted by the lines of sight [7]. Therefore, for standard DC operation the carbon signals represent average quantities, not localized events. Only in case of oscillation of the magnetic structure at the $2 \mathrm{~Hz}$ (see Section 3) channel 4 intercepts one foot print of the magnetic disturbance. For 3/1 DED DC operation, channels 2 and 3 intercept the foot print of the strike zones, while the others are far from localized events. Two further channels (channels A and B in Fig. 1) have been added at the end of the 12/4 campaign (hence available only during the $3 / 1$ campaign) for the detection of CIII lines emitted in the vicinity of and on the ALT limiter. In this study, we make comparison between our UV carbon signals and VUV carbon signals measured at a different location in toroidal and poloidal angles. The relative location of the two diagnostics is such that, in general, the helical structure of the disturbance does not correlate the signals of the two diagnostics, except with 12/4 operation, for which channel 2 of the UV diagnostic sees the same foot print seen by the VUV diagnostic.

\section{Experimental data}

3.1. $m=12, n=4$ mode

When the DED is operated in $12 / 4$ mode, the magnetic surface $q=3$ is positioned as close as possible to the DED coils. This shift is followed by the onset of 


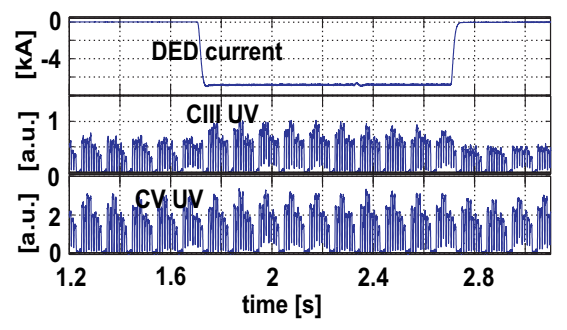

Fig. 2. TEXTOR shot no. 93087. Time traces (from the top) of the DED current and of the CIII and CV signals in UV for DED DC 12/4 operation.

the DED currents. In spite of the marked effects induced on the edge magnetic structure and on the recycling at the four stripes of the strike zones (see Ref. [2]), the influence of DED on carbon signals measured far from the stripes (see Section 2) is not very pronounced. CI and CII signals in the visible, measured on the equatorial plane of the divertor targets on the HFS, increase by about $20-30 \%$, as well as the CIII signal measured in the VUV. Comparable behavior is observed for our UV CIII signals measured along the eight chords. CV remains stationary both for the VUV line and for the eight channel diagnostic (see Fig. 2). The spectroscopic signals from the ALT toroidal limiter on the LFS are generally not available, due to the shift of the plasma column toward the HFS prior to the onset of the DED currents. However, indirect information on the total impurity fluxes can be derived from bolometric measurements (four cameras), which indicate only a minor increase in the total radiated power at the onset of DED currents. Bremsstrahlung signals are consistent with a slight increase of $Z_{\text {eff. }}$ In one series of discharges with DED currents $I_{\mathrm{DED}}=7 \mathrm{kA}$ in which the strike points were swept

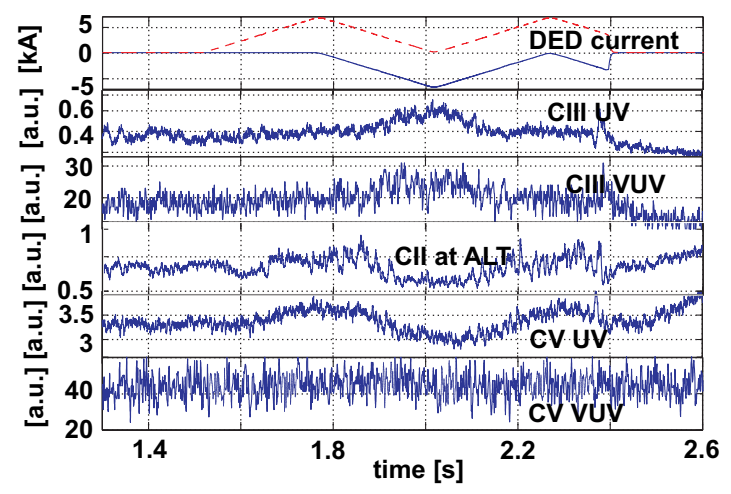

Fig. 3. TEXTOR shot no. 92847. Time traces (from the top) of the DED currents, CIII signal UV on channel 4, CIII signal VUV, CII on ALT, CV in UV on channel 4, CV in VUV for DED $12 / 4$ mode at $2 \mathrm{~Hz}$ oscillation. at a frequency of $2 \mathrm{~Hz}$, the eight channel diagnostic was operated in continuous mode (see Section 2) with CIII and CV signals from channel 4 . The UV CIII signal increases by more than $50 \%$ while $\mathrm{CV}$ signal oscillates moderately (see Fig. 3). The VUV signals show the increase of CIII by about $30 \%$ and the constancy in the $\mathrm{CV}$ line. The different response of the two diagnostics derives from the lack of correlation via the helical structure (VUV is far from the stripes and channel 4 of the UV is on one stripe). In this case, VUV represents the global response of CIII and CV, while UV represents the local response to the action of the magnetic perturbation.

\section{2. $m=3, n=1$ mode}

Since in this configuration the error field penetrates deeper into the plasma, there is no need for shifting the plasma column toward the HFS. Due to the presence of a strong Fourier component $m=2$ in the $3 / 1$ spectrum of DED on TEXTOR (see Ref. [8]), at a certain value of $I_{\mathrm{DED}}$, which is about $0.8 \mathrm{kA}$ for DC operation and nearly a factor of two higher for AC, the 2/1

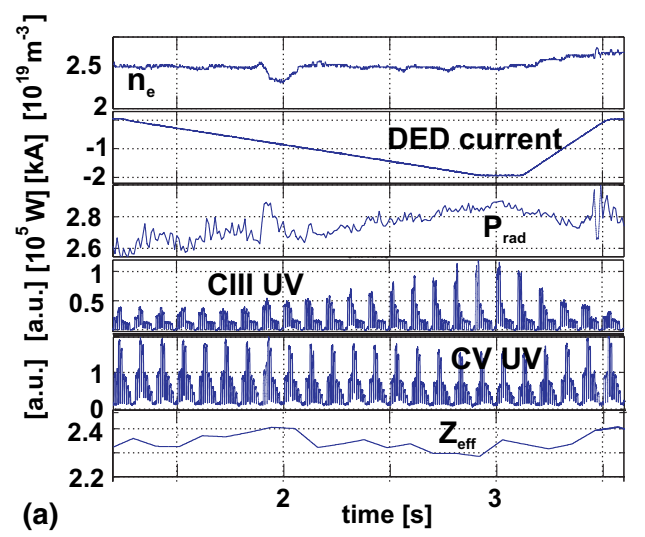

(b)

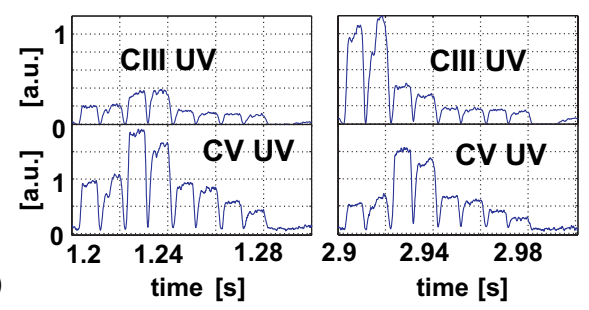

Fig. 4. (a) TEXTOR shot no. 94454. Time traces (from the top) of the central line average density, DED current, $P_{\text {rad }}$, CIII and $\mathrm{CV}$ in the UV, and average $Z_{\text {eff }}$ for DED DC $3 / 1$ operation. At about $t=1.9 \mathrm{~s}$ the $2 / 1$ tearing mode locks to the field. (b) TEXTOR shot no. 94454. Intensity of CIII and CV signals in the eight channels at DED current $I_{\mathrm{DED}}=0$ and at $I_{\mathrm{DED}}=2 \mathrm{kA}$ for 3/1 operation. Channel 2 appears first and channel 8 last in the series of eight signals. 
mode locks to the error field. This locking is correlated with a noticeable increase in plasma-wall interaction and loss of particles and energy from the plasma. Typically (see Fig. 4(a)), in $I_{\text {DED }}$ current up experiments the carbon lines remain nearly unchanged up to the locking of the $2 / 1$ mode. From this time on, the total radiated power, $P_{\text {rad }}$ and the CIII signals from the eight channel diagnostic increase in time, while simultaneously the CV signals decrease. The two CIII signals on the LFS decrease; $Z_{\text {eff }}$ tends to decrease only slightly, but systematically in all discharges. CVI signals from CXRS are consistent with a reduction of about $20 \%$ of the carbon density in the plasma core [9]. The level of the increase of the CIII signals from the eight channel diagnostic, as well as that of decrease of the $\mathrm{CV}$ signals is, however, quite different for different channels. As can be seen in a zoom of the CIII and CV signals (see Fig. 4(b)), the CIII line increases by a factor of 5 in the first two channels (channels 2 and 3) while increases only about by $10 \%$ on the other cannels. Similar, but opposite in sign, is the behavior of $\mathrm{CV}$ line, which decreases by nearly a factor of two on channels 2 and 3 and only by about $10 \%$ on the others.

\section{Discussion}

To a very first approximation one can assume the fluxes of $\mathrm{C}^{3+}$ ions to be proportional to CIII signal (emitted by $\mathrm{C}^{2+}$ ions) and the fluxes of $\mathrm{C}^{5+}$ ions to be proportional to the $\mathrm{CV}$ signal (emitted by $\mathrm{C}^{4+}$ ions). Indeed, although the ionizations per photon for the CIII line and for the CV line are function of the electron temperature [10], in the presence of temperature gradients, as in the plasma edge of tokamaks, the ions of any given ionization level tend to be located at a radial position in which the value of the electron temperature is about one half of their ionization potential [11]. This means, for example in the case of CIII line, that the ratio between the number of photons emitted by $\mathrm{C}^{2+}$ ions (the CIII signal) and the number of ionization events from $\mathrm{C}^{2+}$ ions to $\mathrm{C}^{3+}$ ions (the flux of $\mathrm{C}^{3+}$ ions) can be assumed to be constant. For DC 12/4 operation one has to compare the average increase with the onset of DED of about 25 $30 \%$ of CIII signals in the area of the divertor plate in the HFS with the decrease of the signals in the area of the toroidal limiter ALT in the LFS, which is assumed to be of similar level. Considering the ratio of the surface of the divertor to the surface of the ALT limiter (about 2) one arrives at a modest total increase of $C^{3+}$ fluxes of about $10-15 \%$. This rough estimate is consistent with the observed marginal increase of $P_{\text {rad }}$, which is related essentially to the densities of Li-like $\mathrm{C}^{3+}$ ions. Apart from the small oscillation observed on the channel 4 for the $2 \mathrm{~Hz}$ experiment, the flux of the $\mathrm{C}^{5+}$ ions generally does not change in the channels of the UV diagnos- tic as well as for the VUV diagnostic for DC operation. We can conclude, therefore, that the ratio of the CIII to CV signals increases slightly, in average, for 12/4 DED mode. Since the $\mathrm{C}^{3+}$ ions are located in the edge perturbed zone of the DED field, while $\mathrm{C}^{5+}$ ions are located in the unperturbed zone well inside the $q=3$ surface, the change of the ratio CIII/CV can be taken as a qualitative figure of merit for the screening efficiency of the DED field. The higher becomes this ratio the better is the DED screening efficiency.

In 3/1 mode DC operation of the DED, when the 2/1 tearing mode becomes locked a localized extremely relevant event occurs in correspondence of channels 2 and 3 of the UV diagnostic (see Fig 4 and Fig 4(a)). In fact, channels 2 and 3 intercept the foot print of the strike zones of the $3 / 1$ structure generated by the DED currents [12]. While on these two channels the ratio CIII/ $\mathrm{CV}$ increases up to a factor of 10 for DED currents up to $2 \mathrm{kA}$, see Fig. 5, on the other channels this ratio increases up to a factor of two. This difference makes a clear distinction between local events and global response of the plasma. In line with the interpretation of the action of the Ergodic Divertor of Tore Supra on impurities [4] given in Ref. [5], the experimental findings of the DED effects can be explained as follows. The $m / n=3 / 1$ field structure generated by DED (long decay length, see Section 1) connects the plasma core with the edge plasma and causes a plasma flow along field lines. Due to frictional forces (charge dependent), highly ionized impurities are selectively dragged and expelled, resulting in a global plasma decontamination of nonrecycling impurities. On the other hand, the low ionized carbon ions, which are mostly responsible for the total radiated power, are hardly affected by this expulsion mechanism. Modeling of carbon transport made with the 2D fluid code TECXY [13], based on the transport mechanism used in [4], reproduces the main features of the experimental data reported in this paper.

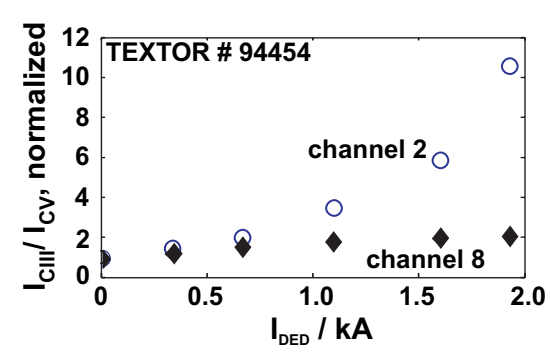

Fig. 5. The ratio CIII/CV (normalized for $I_{\mathrm{DED}}=0$ ) as a function of $I_{\mathrm{DED}}$ for channel 2 pointing at the zone of strong local plasma-wall interaction, and for channel 8. 


\section{Conclusion}

Although for 12/4 DED mode of operation the ratio CIII/CV tends to increase slightly, it would be premature to asses the existence of a carbon screening effect. This loss of screening might depend on the low penetration together with the low level of the radial magnetic field strength generated so far by the DED currents.

In 3/1 DED mode, plasma decontamination of highly ionized carbon ions has been clearly identified. This decontamination, which does not involve low ionized carbon ions, hence the power radiated, can be explained in terms of plasma flow along field lines and frictional forces. This mechanism of plasma decontamination of highly ionized non-recycling ions is, however, associated with losses on the particle and energy channels.

\section{Acknowledgments}

The support by the FWO (Fund for Scientific Research-Flanders) is acknowledged.

\section{References}

[1] K.H. Finken et al., Nucl. Fus. 38 (1998) 515

[2] M. Lehnen et al., these Proceedings. doi:10.1016/j.jnucmat. 2004.10.100.

[3] S.S. Abdullaev et al., Phys. Plasmas (6) 153.

[4] Ph. Ghendrih et al., Plasma Phys. Control. Fus. (1996) 1653.

[5] M.Z. Tokar et al., Plasma Phys. Control. Fus. 39 (1997) 569.

[6] K. Crombé et al., 29th EPS Conference Montreux (2002), ECA Vol. 26B, P1-2.056.

[7] W. Jakubowski et al., Nucl. Fus. Vol. 6 (44) (2004).

[8] Y. Liang et al., paper P1-126 presented at the 31st EPS Conference, London, 2004.

[9] M. Von Hellermann et al., paper P-118 presented at the 31st EPS Conference, London, 2004.

[10] A. Pospieszczyk, in: R.K. Janev, H.W. Drawin (Eds.), Atomic and Plasma-Material Interaction Processes in Controlled Thermonuclear Fusion, Elsevier Science B.V., 1993.

[11] G. Telesca et al., Nucl. Fus. 34 (1994) 625.

[12] S.S. Abdullaev, private communication.

[13] H. Gerhauser, Zagórski, et al., these Proceedings. doi:10.1016/j.jnucmat.2004.07.059. 